\title{
Impacto de la educación sanitaria escolar, hacinamiento y parasitosis intestinal en niños preescolares
}

\section{Impact of school health education, bowling and intestinal parasitosis in preschool children}

\author{
Zuta Arriola Noemi ${ }^{1}$, Rojas Salazar Arcelia Olga $^{2}$, Mori Paredes Manuel Alberto ${ }^{3}$, Cajas Bravo Verónica ${ }^{4}$ \\ ${ }^{1}$ Universidad Nacional del Callao, e-mail: noezut-unac@hotmail.com \\ ${ }^{2}$ Universidad Nacional del Callao, e-mail: m2arcelia@hotmail.com \\ ${ }^{3}$ Universidad César Vallejo, e-mail: m2paredes@hotmail.com \\ ${ }^{4}$ Universidad Hermilio Valdizán Huánuco, e-mail: veronica-cajas@hotmail.com
}

\author{
Recibido e1: 19/10/2018 \\ Aceptado e1: 28/12/2018
}

\section{RESUMEN}

La presente investigación estudió el impacto de la enteroparasitosis en niños y analiza el papel de la educación sanitaria en la promoción de la salud en niños y familiares del Centro Educativo Inicial Estatal "Paz y Amor" de la Región Callao (Perú), durante el año 2017. El estudio se enmarca dentro de una investigación de tipo descriptivo transversal. La muestra estuvo conformada por 120 niños de 3 a 5 años, de los cuales se recolectaron muestras de heces fecales para conocer la prevalencia de parasitosis intestinal, se aplicó un instrumento de encuesta directa a los padres sobre las condiciones socioeconómicas. Así mismo se realizaron charlas educativas de promoción de la salud en la población de estudio antes y después de la recolección de muestras fecales. Para el análisis estadístico se utilizaron pruebas de chi cuadrado y técnicas descriptivas. Destacan como resultado la significancia estadística entre el número de niños en casa y el número de personas que duermen en cama $(p<0.05)$ con presencia de parasitosis intestinal (Enterobius vermicularis).

Palabras clave: Hacinamiento, educación sanitaria, parasitosis intestinal, enteroparásitos.
ABSTRACT

The present investigation studied the prevalence of enteroparasitosis in children and analyzes the role of health education in the promotion of health in children, relatives and teachers of a State Initial Educational Center of the Callao Region (Peru). The study is part of a transversal descriptive research. The sample consisted of 120 children from 3 to 5 years of age, from whom stool samples were collected to know the prevalence of intestinal parasitosis, a direct survey instrument was applied to parents on socioeconomic conditions. Likewise, educational health promotion talks were held in the study population before and after the collection of faecal samples. For the statistical analysis, chi square tests and descriptive techniques were used. The statistical significance between the number of children at home and the number of people who sleep in bed $(\mathrm{p}<0.05)$ with presence of intestinal parasitosis (Enterobius vermicularis) stand out as a result.

Keywords: Overcrowding, health education, intestinal parasitosis, enteroparasites. 


\section{INTRODUCCIÓN}

Las parasitosis intestinales son muy frecuentes en niños y representan un problema de salud pública. En Latinoamérica, se estima que más de 40 millones de preescolares están expuestos a un tipo de parasitosis intestinal, representando uno de los grandes problemas de salud pública que afectan más del 30\% de la población mundial. La prevalencia e intensidad de las parasitosis están asociadas a mayor riesgo de morbilidad y tienden a ser elevadas principalmente en la población en edad escolar.

En los países subdesarrollados los índices de parasitosis llegan hasta el 90\%, aumentando significativamente según el nivel socioeconómico (Leite Seixas et al., 2012); adicionando a ello, la falta de prevención por una inadecuada educación para la salud que compromete responsabilidades de los padres, instituciones educativas y del Estado.

El diseño de un plan estratégico de educación sanitaria desde la atención primaria de salud es una acción prioritaria en la prevención de la parasitosis. Las parasitosis en niños afectan su desarrollo intelectual y físico. Estudios como el de Reyes \& Betancourt, (2012) y Wang et al., (2018) demostraron que a través de charlas educativas de 48 promoción de salud a los estudiantes, familiares, maestros y trabajadores de Instituciones Educativas permiten la adopción de medidas higiénicosanitarias saludables, lo que influirá positivamente en la disminución de la prevalencia e incidencia de las parasitosis intestinales en la población infantil. Por tanto, la Educación sanitaria escolar puede contribuir efectivamente para el control y prevención de parasitosis, ya que padres de familia, niños y maestros habiendo adquirido conocimientos en las charlas educativas se evita la adquisición de las enfermedades parasitarias y de sus consecuencias, tal como lo demuestran estudios realizados por (Gomes et al., 2016 y Fernández, Estrada, Crespo, \& Rodríguez, 2008).

El presente estudio identificó algunos de los factores socioeconómicos responsables para la prevalencia de parasitosis intestinal en niños, tales como el hacinamiento habitacional. Entendiéndose, como hacinamiento a los límites máximos de densidad poblacional en relación entre el número de habitantes y el espacio disponible (Lentini \& Palero, 1997), lo que conlleva al problema de sobrecarga habitacional, tres o más personas por cuarto destinado a habitación o dormitorio. Que, a nivel de individuo, pueden producir alteraciones en la salud física, al facilitarse la transmisión de enfermedades infecto-contagiosas (Puga, 1979). Por tanto, en la educación sanitaria se debe también focalizar la atención en el hacinamiento como un factor socioeconómico que favorece la prevalencia de parasitosis intestinal en niños que unido a la presencia de animales en viviendas pequeñas, que exige un mayor cuidado en la higiene (Ibáñez, 2004) ya que los comportamientos de higiene adecuados y las condiciones ambientales son fundamentales para la salud, el desarrollo y el bienestar de los niños (Moffa et al., 2019). Diferentes estudios (Altamirano, 2017 y Morales, 2016) han indicado que la aparición de parasitosis es bastante variable en las diferentes regiones del Perú y están relacionados a aspectos socioeconómicos y la falta de educación sanitaria.

En ese escenario el artículo tiene como objetivo evaluar la prevalencia de enteroparasitosis en niños después de la educación sanitaria basada en la identificación de necesidades educativas, así como en la elaboración, ejecución y evaluación de un plan de educación sanitaria; para analizar su papel en la promoción de la salud en niños y familiares del Centro Educativo Inicial Estatal "Paz y Amor" en el Distrito de la Perla, Región Callao (Perú) durante el año 2017.

\section{MARCO TEÓRICO}

\section{Educación Sanitaria / Educación para la Salud}

La prevención de enfermedades parasitarias debe abordarse desde el colegio a través de consejos de higiene alimentaria en programas de educación sanitaria. La Organización Mundial de Salud(OMS) recomienda los tratamientos masivos para luchar 
contra los parásitos intestinales especialmente en el tratamiento de los niños en edad preescolar. Pero, debe tomarse en cuenta que los tratamientos antiparasitarios no son eficaces en la población general si no se acompañan de una educación sanitaria continua ya que existe desconocimiento de la higiene alimentaria. (Bourée, 2016)

En el Perú, el Ministerio de Salud - MINSA, (2011) refiere que:

Es el proceso sociocultural permanente por el cual las personas se van desarrollando para beneficio de sí mismas y de la sociedad, mediante una intervención activa e intencionada en los aprendizajes, que se logran por interacción en ámbitos de educación formal y no formal. Involucra el desarrollo de capacidades $y$ habilidades sociales y personales necesarias para adoptar medidas destinadas a mejorar la propia salud y crear las posibilidades para actuar en la comunidad, logrando cambios que favorezcan la salud colectiva (pág. 17)

Se dice que la educación para la salud es uno de los instrumentos que utiliza la Promoción de la Salud, para facilitar la adopción de actitudes y comportamientos sanos (Chamorro, 2010). Las prácticas educativas bien aplicadas sí llevan a las personas a adquirir conocimientos para la prevención de parasitosis, alcanzando objetivos propuestos y evidenciando el valor de la orientación pedagógica para la concientización de la población (Ferreira \& Andrade, 2005). Entonces las acciones educativas para ser efectivas no deben tener la visión simplista y limitada de "formación" de ciudadanos, deben ser actividades que permitan el intercambio de saberes y de experiencias en la búsqueda de prevenir enfermedades tales como la parasitosis intestinal en niños.

Para adoptar medidas para mejorar la salud y prevenir infecciones tales como las parasitosis, se debe empezar con la implementación de la educación sanitaria en los colegios, tanto para los padres como estudiantes. Para ello es necesario usar medios de difusión masiva para la transmisión de conocimientos a fin de que la mayor cantidad de personas se vean favorecidas con ellos. (Altamirano, 2017)

Ello permitiría un cambio de prácticas en relación con las mascotas y disminuir así el riesgo de exposición a enfermedades zoonóticas. Sin embargo, el problema de parasitosis, no solo radica en resolver la disminución de los parásitos en la población, si no es parte de graves problemas y desigualdades sociales como la pobreza, desnutrición y en especial de los malos hábitos higiénicos, dietéticos y culturales que involucra a la comunidad rural y los más afectados son los niños. La disminución de parasitosis tiene que ir junto con la mejora de calidad de vida de los pobladores, para superar estos problemas.

\section{Parasitosis intestinales en niños}

Las parasitosis infantiles provocan trastornos digestivos, tales como diarreas, cuyo síntoma se manifiesta frecuentemente en la infestación por protozoos. En la infestación por helmintos suele haber una eosinofilia sanguínea (Bourée, 2016). Las parasitosis intestinales infantiles pueden ser asintomáticas si la infección es escasa, o provocar trastornos digestivos y sistémicos graves en caso de infección masiva. El daño que producen los parásitos en los niños depende de la tríada ecológica agente, hospedero y medio ambiente. (Werner Apt, 2014)

Los parásitos intestinales se transmiten de una persona a otra por medio de la vía fecal-oral así por ejemplo Entamoeba histolytica agente causal de amebiasis intestinal aguda y el absceso hepático amebiano. Giardia lamblia (Sín: G. lamblia; G. intestinalis) es el protozoo intestinal flagelado agente causal de la Giardiasis. La Giardiasis es una Zoonosis y puede ser adquirida por la ingestión de agua contaminada con heces animales o humanos portadores. Los quistes de Giardia son muy resistentes al medio ambiente. La profilaxis de estos parásitos se fundamenta en el control adecuado de excretas, la higiene personal y de los alimentos. 
La OMS (1981), considera que la parasitosis es una de las principales causas de morbilidad, ligada a la pobreza y relacionada con inadecuada higiene personal y de los alimentos crudos, falta de servicios sanitarios, falta de provisión de agua potable y contaminación fecal del ambiente. Los parásitos infectan a personas de todas las edades, pero son los niños los más susceptibles, a quienes les causa trastornos en el crecimiento-desarrollo y en el aprendizaje.

La prevalencia y la intensidad de las infecciones del intestino humano por protozoos y helmintos en el mundo presentan variaciones considerables de distribución y aparición estacional a causa de factores geográficos y climáticos y de actividades humanas (como el cambio de ambiente y el mejoramiento del saneamiento). Los factores responsables de una mayor intensidad de las infecciones intestinales por parásitos pueden dividirse en dos grupos: los ambientales y los socioeconómicos. Los factores ambientales son responsables del desarrollo y la difusión de las etapas infectantes del parásito, en tanto que los factores socioeconómicos son responsables de que el ambiente se contamine con parásitos restableciendo contactos con las etapas infectantes del parásito.

50 Lo que se sabe acerca de los factores socioeconómico responsables de la trasmisión de helmintos es superficial y escaso, debido a que hay muchas clases de comportamiento humano que pueden influir sobre la prevalencia y la intensidad de las infecciones intestinales. La mayoría de los estudios emprendidos hasta ahora sobre las infecciones parasitarias intestinales han estado relacionados con determinantes sociales tradicionales, como la edad y el sexo; pocos han estudiado factores relaciones a la densidad de la población, factores profesionales y étnicos.

La relación de la parasitosis con la edad está vinculada con el modo principal de transmisión, los niños pequeños contraen ascariasis jugando en el suelo contaminado alrededor de las casas. Cuando intervienen factores más comunales como el agua, los alimentos o el polvo, como portadores de las fases infectantes, dependen más del estado inmunitario del huésped que de la edad o factores ambientales. Las infecciones intestinales transmitidas por el suelo generalmente son menos comunes en las zonas urbanas que en las rurales o por lo menos no son tan intensivas. La parasitosis tales como amibiasis, guardias e himenolepiasis se transmiten por contacto directo con el agua o alimentos contaminados, estos son comunes en las poblaciones urbanas y suburbanas densamente pobladas (OMS 1981)

Los niños constituyen, la población más susceptible a enfermedades parasitarias debido a su inmadurez inmunológica y al escaso desarrollo de hábitos higiénicos. En ellos, les causa principalmente la disminución del apetito, hinchazón abdominal y diarrea. La parasitosis crónica causa alteraciones en el crecimiento y en el aprendizaje. Por lo tanto, las condiciones de saneamiento ambiental, sumadas a una insuficiente educación sanitaria facilitan su aparición (Zonta et al., 2016). Las condiciones de vivienda pueden repercutir sobre la salud física ya que expone a sus residentes a alérgenos, microorganismos y componentes químicos nocivos para la salud causando reacciones alérgicas $\mathrm{y}$ distintas enfermedades infecciosas. (Novoa et al., 2014)

La presencia de parásitos intestinales, especialmente cuando la carga parasitaria es alta constituye un importante factor de riesgo, si se halla asociado a condiciones de pobreza y desnutrición (Buyasyisqui, Cesani, Haedo, Oyhenart, \& Garbossa, 2009).

\section{METODOLOGÍA}

Se realizó un estudio descriptivo, con una población conformada por 350 niños de 3 , 4, y 5 años matriculados en el año académico 2015 en un Jardín estatal del Distrito de la Perla-Callao, en los turnos mañana y tarde. Se trabajó con una muestra estratificada de 120 niños: 42 niños de 03 años, 35 niños de 04 años, 43 niños de 05 años. 
Para la educación sanitaria se tomaron en cuenta los momentos de la educación Sanitaria propuestos por el Ministerio de Salud (MINSA, 2011): (1) identificación de necesidades educativas en los padres de familia y profesores en la reunión mensual de padres en el colegio. (2) Elaboración del plan de la educación sanitaria y materiales educativos, (3) Ejecución de la educación sanitaria (4) Evaluación del proceso educativo, donde se pudo evidenciar un impacto positivo al fomentar toma de decisiones favorables para la salud.

Previo al inicio de las charlas educativas, se sostuvo una reunión en el colegio con el objetivo de explicar la importancia, objetivos y beneficios del estudio y así accedieran a dar las muestras de heces, previo consentimiento informado firmado por las padres o tutores. Los padres de los niños que aceptaron participar en el estudio recibieron un envase de plástico nuevo, limpio de boca ancha y tapa rosca y dos láminas porta objetos con una cinta scotch (test de Graham).

Las charlas educativas a los padres /o responsables, profesores de los niños, se desarrollaron a través de las técnicas de Exposición oral y debate dirigido, empleándose el análisis de láminas ilustrativas; y se realizaron con el apoyo de los estudiantes del VI ciclo de la Escuela Profesional de Enfermería en reuniones periódicas que el colegio sostuvo con los padres de familia, así mismo se coordinó con el Centro de Salud de la Perla, para que en la educación sanitaria que se brinda se enfatice en el tema de higiene y medio ambiente para la prevención de la parasitosis intestinal. (MINSA, 2011)

Después de un intervalo de dos meses de haber llevado a cabo el proceso de educación sanitaria se procedió a recolectar los datos respecto a la prevalencia de enteroparasitosis en niños para evaluar el impacto que tuvo dicha educación en el cuidado de la salud.

Para el análisis coproparasitológico se aplicó la Técnica de Sedimentación Espontánea en Tubo (TSET) (Fabián, Tello \& Náquira, 2003) para el hallazgo de huevos o quistes de enteroparásitos y la Técnica de la cinta adhesiva para el hallazgo de huevos de Enterobius vermicularis.

Así mismo se aplicó un cuestionario de 20 preguntas a madres/padres de familia de los niños que participaron en el estudio para la identificación de las características habitacionales de la familia.

Con los datos recolectados se realizaron análisis descriptivos y significancia estadística de la asociación con características habitacionales, utilizándose el paquete estadístico STATA V. 12 y Excel.

\section{RESULTADOS Y DISCUSIÓN}

Para los exámenes coproparasitológicos se analizó un total de 120 muestras de heces de niños, se encontró que 65 muestras analizadas (54.2\%) presentaron algún tipo de parásito

Tabla 1

Presencia de Parasitosis intestinales en niños de la I.E - Callao

\begin{tabular}{lll}
\hline & Frecuencia & Porcentaje \\
\hline NO & 55 & 45,8 \\
SI & 65 & 54,2 \\
Total & 120 & 100,0 \\
\hline \multicolumn{2}{l}{ Fuente: Elaboración propia }
\end{tabular}

De los parásitos hallados en el estudio, 49 muestras $(40.8 \%)$ correspondieron a Enterobius vermicularis, 12 (18,8\%) Giardia duodenalis, 38 (59.3\%) Entamoeba coli y 14 (22\%) Endolimax nana en las heces de los niños de 3 a 5 años (Tabla $\mathrm{N}^{\circ} 2$ ). La frecuencia de parasitosis intestinal fue bastante elevada en la población estudiada (54.2\%).

De forma coincidente, los resultados de Rodríguez, et.al (2010) demuestran la alta frecuencia de enteroparasitosis entre los niños de las comunidades de Cajamarca, lo cual sería consecuencia de las condiciones de vida precarias, los inadecuados hábitos higiénicos y el hacinamiento humano en que viven (Marcos, et.al., 2002). Coincidiendo con otros estudios (Devera, Mago \& Rumhein, 2006 y Rivera, López, Rodríguez y Ulloa, 2008), Giardia lamblia es el parásito patógeno de mayor 
predominio entre los niños, que puede causar pérdida de peso y afectar el rendimiento escolar de los mismos (Çeliksöz, et.al., 2005).

\section{Tabla 2}

Prevalencia de parasitosis en niños preescolares de una Institución Educativa

\begin{tabular}{cc}
\hline Tipo de parasito & Prevalencia \\
\hline Giardia lamblia & $12(18.8 \%)$ \\
Entamoeba coli & $38(59.3 \%)$ \\
Endolimax nana & $14(22 \%)$ \\
Enterobius vermicularis & $49(40.8 \%)$ \\
Total & $64(100 \%)$ \\
\hline Fuente: Elaboración propia &
\end{tabular}

Es preocupante la presencia de Giardia lamblia (18.8\%), el cual es un parásito que habita en el duodeno del niño, agente causal de la Giardiasis, y es adquirido por la ingestión de agua contaminada con heces de animales o humanos portadores (Brock \& Madigan, 2015), su presencia en los niños causa malabsorción intestinal y diarreas, que redundan en una malnutrición intestinal y bajo rendimiento académico. Es importante considerar la transmisión fecal-oral del parásito de un niño a otro en los centros educativos y de los niños infectados a otros miembros de la familia. (Black, Dykes, Sinclair, \& Wells, 1977)

Diferentes estudios (Raymundo, et.al., 2013; Jiménez et.al., 2011 y Pino, 2016) reportan similares índices de parasitosis intestinal en 52 escolares de diferentes colegios públicos a nivel nacional. Los resultados reflejan las deficientes condiciones de saneamiento básico, la presencia de animales en casa, el hacinamiento, la calidad de agua e higiene de los estudiantes tanto en la escuela como en la casa. Por tanto, en la educación sanitaria se incluyeron temas de higiene en la vivienda y en la escuela, así como higiene personal para la prevención de la parasitosis.

En este estudio, el 55\% de las familias de los niños conviven con tres a cuatro adultos y el $33 \%$ con más de cuatro adultos en la familia. El número máximo de personas que duermen por cama es mayor a 2 en un $30 \%$ y el número de niños en casa es mayor de 2 en un $29 \%$, el $58 \%$ vive en casa independiente, el $20 \%$ en departamento. Asimismo, el 99\% cuentan con un solo servicio higiénico en su casa y el 78.3\% tienen animales en casa (Tabla 3)

Tabla 3

Condiciones de las viviendas y convivencia de las familias de los pre-escolares

\begin{tabular}{|c|c|c|}
\hline VARIABLE & CATEGORIA & $\begin{array}{l}\text { Frecuencia } \\
\text { (\%) }\end{array}$ \\
\hline \multirow{3}{*}{$\begin{array}{l}N^{o} \text { de adultos en vivienda } \\
\text { de los niños }\end{array}$} & Menor de 3 & $32(26.7 \%)$ \\
\hline & De 3 a 4 & $55(45.8 \%)$ \\
\hline & Mayor de 4 & $33(27.5 \%)$ \\
\hline \multirow[t]{2}{*}{$N^{o}$ de niños en vivienda } & Menor o igual a 2 & $91(75.8 \%)$ \\
\hline & Mayor de 2 & $29(24.2 \%)$ \\
\hline$N^{o}$ máximo de personas & Menor o igual a 2 & $84(70 \%)$ \\
\hline \multirow[t]{2}{*}{ que duermen por cama } & Mayor de 2 & $36(30 \%)$ \\
\hline & No tiene seguro & $25(20,8 \%)$ \\
\hline \multirow[t]{5}{*}{$\begin{array}{l}\text { Tipo de vivienda de los } \\
\text { niños }\end{array}$} & $\begin{array}{l}\text { Casa en condominio } \\
\text { o residencial }\end{array}$ & $5(4.2 \%)$ \\
\hline & Casa independiente & $58(48,3 \%)$ \\
\hline & En quinta & $14(11,7 \%)$ \\
\hline & En departamento & $24(20 \%)$ \\
\hline & Cuartos & $19(15,8 \%)$ \\
\hline servicios & 01 & $119(99,2 \%)$ \\
\hline \multirow[t]{2}{*}{ higiénicos en las viviendas } & 02 & $1(0,8 \%)$ \\
\hline & 03 & $0 \%$ \\
\hline \multirow{2}{*}{$\begin{array}{l}\text { Presencia de animales en } \\
\text { la vivienda }\end{array}$} & $\mathrm{Si}$ & $94(78,3 \%)$ \\
\hline & No & $26(21,7 \%)$ \\
\hline
\end{tabular}

Fuente: Elaboración propia

El número máximo de personas que duermen en una sola cama es mayor a dos personas, así mismo en el $27 \%$ de familia existen adultos en número mayor a 4 , estas condiciones son consideradas por el INEI (2007) como vivienda hacinada si este cociente es mayor a 3, es decir, el grado de hacinamiento es un índice que clasifica a las viviendas como viviendas con hacinamiento cuando el número de personas por habitación es mayor a 3 .

Los principales indicadores relacionados con la salud de las familias en su domicilio para propiciar viviendas saludables son promovidos por la Organización Panamericana de la Salud (Santa María, 2008). La Organización Mundial de la Salud ha tratado el tema relacionado a la vivienda saludable, aludiendo que una parte de los factores necesarios para protegerse contra las enfermedades transmisibles es el espacio vital y que son especialmente vulnerables a las condiciones de la vivienda y sus efectos en la salud, las mujeres, niños, ancianos, los enfermos crónicos y los discapacitados (Organización Panamericana de la Salud - CEPIS, 1999) 
Por tanto, la presencia de algún tipo de parásito en niños menores de 5 años de edad está fuertemente influenciada por el hacinamiento y la falta de higiene. En nuestro estudio se halló una presencia significativa de parasitosis en niños, así como un gran número de personas que duermen en cama en proporción al número de niños que viven en casa $(\mathrm{p}<0.05)$ (Tabla 4 y 5$)$.

\section{Tabla 4}

Número máximo de personas que duermen por cama y parasitosis intestinal en preescolares

\begin{tabular}{llll}
\hline $\begin{array}{l}\text { PARASITOSIS } \\
\text { INTESTINAL }\end{array}$ & $\begin{array}{l}\text { Número máximo de personas } \\
\text { que duermen por cama: }\end{array}$ & $\begin{array}{l}\text { Sig } \\
(\mathrm{p})\end{array}$ \\
\cline { 2 - 4 } & menor o igual a 2 & mayor de 2 & \\
\hline NO & 57 & 14 & 0,003 \\
SI & 27 & 22 & \\
TOTAL & $\mathbf{8 4}$ & $\mathbf{3 6}$ & \\
\hline
\end{tabular}

Fuente: Elaboración propia

Tabla 5

Número niños en casa y parasitosis intestinal en preescolares

\begin{tabular}{llllll}
\hline Presencia de parásitos & \multicolumn{4}{c}{$\mathrm{N}^{\mathrm{o}}$ niños en casa } & \multirow{2}{*}{ Sig (p) } \\
\cline { 2 - 5 } & 1 & 2 & 3 & 4 & \\
\hline NO & 41 & 20 & 7 & 3 & 0.000 \\
SI & 10 & 18 & 16 & 5 & \\
Total & 51 & 38 & 23 & 8 & \\
\hline
\end{tabular}

Fuente: Elaboración propia

La falta de educación sanitaria, asociada a las precarias condiciones socioeconómicas en que viven las familias propicia la presencia de enfermedades y conlleva a la alta prevalencia de parasitismo intestinal en escolares provenientes de áreas rurales. (Barazarte y Quintero, 2001)

Una experiencia en la escuela de educación sanitaria en una organización educativa, se realizó con 35 profesores (Fontana, 2008) a fin de brindar oportunidades de espacios para la reflexión de las condiciones sanitarias, y que la educación sea una herramienta que facilite intervenir con eficacia en acciones de prevención de daños a la salud colectiva e individual.

Asimismo, la investigación de Román, et al., (2014) evidencia que los trabajos desarrollados con paquetes educativos refuerzan y permiten que se incremente el nivel de información sobre la prevención de parasitosis intestinal. Tales resultados fueron similares a los obtenidos por Abril et al. (2009) con población mexicana en Hermosillo, Sonora y Cuernavaca.

La Educación sanitaria escolar tuvo un impacto positivo en la Institución Educativa con la participación de madres de familia de los preescolares, desarrollando la temática de saneamiento básico, presencia de animales en casa, hacinamiento, calidad de agua e higiene de los estudiantes y la descripción de causas, síntomas de las enfermedades parasitarias, ciclo biológico de los parásitos más importantes encontrados en los niños. La Educación sanitaria se desarrolló a través de la técnica de Exposición oral y debate dirigido, empleándose el análisis de láminas ilustrativas, donde los participantes tuvieron la oportunidad de manifestar sus inquietudes sobre el tema.

La Educación sanitaria desarrollada con padres de familia en las sesiones educativas permitieron la enseñanza-aprendizaje, el aprehender a aprender y el desarrollo de competencias y habilidades para la adopción de prácticas higiénicas y hábitos saludables en la comunidad escolar. Se busca lograr que la persona aprenda y modifique sus actitudes (Fishbein y Azjen, 2010) para el desarrollo de conductas que promuevan la salud.

Es importante reportar dificultades vinculadas a la poca participación de padres de familia en cuanto a la prevención de parasitosis. Esto se explica debido a que el perfil cultural marca la relación de los padres con la escuela, donde se asume a la educación desde una responsabilidad más individual que colaborativa y/o participativa (Colás \& Contreras, 2013)

Cabe resaltar que la educación sanitaria y la comunicación socialsonlas estrategias primordiales para la prevención y la promoción de la salud, en la cual deben participar toda la comunidad educativa (MINSA, 2011) para la adopción de hábitos saludables evitando la prevalencia e incidencia de las parasitosis intestinales en la población infantil (Reyes \& Betancourt, 2012). 
Este tema debe considerarse como una prioridad de salud pública y educativa para la prevención; demanda de programas de salud escolar efectivos que incluyan educación y exámenes de salud periódicos, considerando que las acciones en la educación en salud exigen un costo menor, comparado al tratamiento de la enfermedad, por ello la importancia en la mejora de la calidad de vida de las personas. Por tanto, es necesario mencionar que la promoción de la salud en el ámbito escolar es una prioridad impostergable. Asegurar el derecho a la salud y la educación de la infancia es responsabilidad de todos y es la inversión que cada sociedad debe hacer para generar a través de la capacidad creadora y productiva de los jóvenes, un futuro social y humano sostenible.

\section{CONCLUSIONES}

Estos resultados confirman la prevalencia de parasitosis intestinal presente en los niños pre escolares del jardín escolar de la Perla, Callao, por lo que es necesario mejorar el nivel educación sanitaria de escuelas y fomentar el concepto de responsabilidad individual y colectiva en las viviendas de las familias.

Las condiciones de servicio higiénico y la presencia de animales en casa representan factores adversos 54 que promueven la incidencia de parasitosis intestinales al no observarse las medidas higiénicas necesarias. Asimismo, la prevalencia de parásitos en niños menores de 5 años afecta a todos los niños por igual sin distinción de edad ni sexo.

Se resalta el impacto positivo de la educación sanitaria en la Institución Educativa ya que su objetivo es incidir sobre los comportamientos, percepciones, creencias y prácticas de la gente. Por lo tanto, representa una actividad importante para la adopción de hábitos saludables y así evitar o disminuir la prevalencia e incidencia de las parasitosis intestinales en la población infantil. Finalmente, cabe resaltar que, para ser efectiva, la educación sanitaria debe responder a las necesidades particulares de la población a la que va dirigida.

\section{CONFLICTOS DE INTERÉS}

Los autores declaran no tener conflicto de intereses. La Universidad Nacional del Callao (Perú), con la investigación científica promueve el sentido de responsabilidad social, en función de las necesidades de la región Callao.

\section{AGRADECIMIENTO}

Agradecimiento a la Institución Educativa Inicial de la Perla- Callao (Perú) y la Universidad Nacional del Callao por el financiamiento de la presente investigación.

\section{REFERENCIAS BIBLIOGRÁFICAS}

Abril, E. et al. (2009) Promoción de hábitos alimentarios saludables en una escuela primaria de Hermosillo, Sonora, México. Revista de Salud Pública y Nutrición, 10(1).

Altamirano, F. (2017). Factores de riesgo asociados a parasitismo intestinal en niños pre escolares atendidos en el Aclas San Jerónimo. Andahuaylas (Tesis grado de maestro) Universidad Peruana Cayetano Heredia. Recuperado de http://repositorio. upch.edu.pe

Barazarte Y. y Quintero M. (2001) Profilaxis de la Parasitosis Intestinal en la Escuela Rural. Enfoque Constructivista. (Tesis de Pregrado) Universidad de Andes. Núcleo Universitario "Rafael Rangel".

Black, E., Dykes, C., Sinclair, P., \& Wells, G. (1977). Giardiasis in day-care centers: evidence of person-to-person transmission. Pediatrics, 60(4), 486-491.

Bourée, P. (2016). Parasitosis intestinales infantiles. EMC - Tratado de Medicina, 20(4), 1-10. https://doi.org/10.1016/S16365410(16)80667-4

Brock, T. D., \& Madigan, M. T. (2015). Biología de los microorganismos. Madrid: Pearson Educación.

Buyasyisqui, M., Cesani, M., Haedo, A., Oyhenart, E., \& Garbossa, G. (2009). Enteroparasitosis y Desnutrición en una población infantil 
del Norte Argentino. (Tesis de maestría) En: $\quad$ http://www.fcnym.unlp.edu.ar/aabra/ Actas2009/Buyayisqui\%20et\%20alpdf

Çeliksöz A, Aciöz M, Degerli S, Çinar Z, Elaldi N, Erandaç M. (2005). Effects of giardiasis on school success, weight and height indices of primary school children in Turkey. Pediatr Int; 47: 567-571.

Chamorro, R. (2010). Educación para la Salud en el Currículo Escolar: La experiencia de la Institución Educativa 7059 «José Antonio Encinas Franco». Pamplona Alta. Distrito de San Juan de Miraflores-UGEL 01 (Tesis maestría) Pontificia Universidad Católica del Perú. Recuperado de http://tesis.pucp.edu.pe/ repositorio

Colás, P, \& Contreras, J. (2013). La participación de las familias en los centros escolares de Educación Primaria. Revista de Investigación Educativa, 31(2), 499. https:// doi.org/10.6018/rie.31.2.171031

Devera R, Mago Y, Rumhein FA. (2006) Parasitosis intestinales y condiciones socio-sanitarias en niños de una comunidad rural del Estado Bolívar, Venezuela. Rev Biomed; 17: 311-313.

Fabián M, Tello R, \& Náquira C. (2003). Manual de procedimientos de laboratorio para el diagnóstico de los parásitos intestinales del hombre. (INS). Recuperado de http://bvs. minsa.gob.pe/local/INS/165_NT37.pdf

Fernández,H,Estrada, I,Crespo, Y., \& Rodríguez,K. (2008). Intervención educativa para el control del parasitismo intestinal en adolescentes. Revista Archivo Médico de Camagüey, 12(4), 0-0. Recuperado de http://scielo.sld.cu/ scielo.php? script $=$ sci abstract\&pid $=\mathrm{S} 1025$ $\underline{02552008000400004 \& \operatorname{lng}=\mathrm{es} \& \mathrm{nrm}=\mathrm{iso} \& \mathrm{t} \ln }$ $\mathrm{g}=\mathrm{es}$

Fishbein, M. y I. Ajzen (2010). Predicting and changing behavior: The reasoned action approach. New York, Psychology Press (Taylor \& Francis).

Fontana, R. T. (2008). A vigilância sanitária no contexto escolar: um relato de experiência. Revista Brasileira de Enfermagem, (1), 131. $\quad$ https://doi.org/10.1590/S003471672008000100022
Gomes, C., Rodrigues, R., Silva, B., Arruda, A., Silva, N., Macedo, R.., Ferreira, I. (2016). Educação em saude como instrumento de prevenção das parasitoses intestinais no município de Graja- MA. Pesquisa en foco, 21(1). https://doi.org/10.18817/pef. v21i1.1123

Jiménez, J., Vergel, K., García, M.., Vega, F., Uscata, R., Romero, S., Náquira, C. (2011). Parasitosis en niños en edad escolar: relación con el grado de nutrición y aprendizaje. Horizonte Médico, 11(2), 65-69. Recuperado de http://www.horizontemedicina.usmp.edu. pe/index.php/horizontemed/article/view/117

Leite, M, Souza, J., Da Paixão, R., Aquino, M., \& Soares, M. (2012). Avaliação da frequência de parasitos intestinais e do estado nutricional em escolares de uma area periurbana de Salvador, Bahia, Brasil. Revista de Patologia Tropical, 40(4). https://doi.org/10.5216/rpt. v40i4.16762

Lentini, M., \& Palero, D. (1997). El hacinamiento: la dimensión no visible del déficit habitacional. Revista INVI, 12(31). https:// doi.org/10.4067/invi.v12i31.220

Marcos L., Maco V., Terashima A., Samalvides F., Gotuzzo E. (2002). Prevalencia de parasitosis intestinal en niños del valle del Mantaro, Jauja, Perú. Rev Med Hered; 13(3): 85-90

Ministerio de Salud. (2011). Documento Técnico 5 Modelo de Abordaje de Promoción de la Salud en el Perú. Recuperado de http://bvs.minsa. gob.pe/local/promocion $/ 203$ prom $30 . \mathrm{pdf}$

Ministerio de Salud. (2011). Guía Técnica de Gestión de Promoción de la Salud en Instituciones Educativas para el Desarrollo Sostenible. Recuperado de http://www. minsa.gob.pe/bvsminsa.asp

Moffa, M., Cronk, R., Fejfar, D., Dancausse, S., Padilla, L. A., \& Bartram, J. (2019). A systematic scoping review of hygiene behaviors and environmental health conditions in institutional care settings for orphaned and abandoned children. Science of The Total Environment, 658, 1161-1174. https://doi.org/10.1016/j. scitotenv.2018.12.286 
Morales, J. (2016). Parasitosis intestinal en preescolares y escolares atendidos en el centro médico EsSalud de Celendín, Cajamarca (Tesis de maestría) Universidad Norbert Wienner. Recuperado de http://repositorio. essalud.gob.pe/handle/ESSALUD/285

Navone G., Gamboa M., Oyhenart E., Orden B.. (2016) Parasitosis intestinales en poblaciones Mbya-Guaraní de la Provincia de Misiones. Aspectos epidemiológicos y nutricionales. Cad Saude Publica; 22:109-18.

Novoa, A., Bosch, J., Díaz, F., Malmusi, D., Darnell, M., \& Trilla, C. (2014). El impacto de la crisis en la relación entre vivienda y salud. Políticas de buenas prácticas para reducir las desigualdades en salud asociadas con las condiciones de vivienda. Gaceta Sanitaria, 28, 44-50. https://doi. org/10.1016/j.gaceta.2014.02.018

Organització Mundial de la Salut. (1981). Infecciones intestinales por protozoos $y$ helmintos: informe de un grupo cientifico de la OMS. Ginebra: Organización Mundial de la Salud.

Organización Panamericana de la Salud (CEPIS). (1999). Documento de Referencia OPS sobre Políticas de Salud en la Vivienda [CEPIS]. Recuperado 27 de abril de 2019, de http:// www.bvsde.paho.org/bvsasv/e/iniciativa/ posicion/posicion.html

Pino, J. R. M. D. (2016). Parasitosis intestinal en preescolares $\mathrm{y}$ escolares atendidos en el centro médico. Horizonte Médico, 16(3), 35-41. Recuperado de http://www. horizontemedicina.usmp.edu.pe/index.php/ horizontemed/article/view/468

Puga, J., (1979) Seminario Vivienda para los Sectores de Menores Ingresos (Eds.). Consecuencias sociales del deficit habitacional en los sectores urbanos de mínimo ingreso. Santiago, Chile: CPU.

Raymundo, A., Flores, M., Iwashita, T., Cuba, S., \& Herencia, E. (2013). Prevalencia de parasitosis intestinal en niños del valle del Mantaro, Jauja, Perú. Revista Medica Herediana, 13(3). https://doi.org/10.20453/ rmh.v13i3.695
Reyes, I., \& Betancourt, O. (2012). Parasitosis intestinal y educación sanitaria en alumnos de la Unidad Educativa Guamacho. Revista Cubana de Investigaciones Biomédicas, 31(1), 123-128. Recuperado en 24 de mayo de 2019, de http://scielo.sld.cu/ scielo.php?script $=$ sci arttext\&pid $=\mathrm{S} 0864$ 03002012000100013\&lng=es\&tlng=es.

Rivera M., López, J., Rodríguez, C. (2008) Enteroparasitosis infantil en guarderías de la zona rural de Cajamarca. Rev Peru Med Exp Salud Publica; 25(4): 445-446.

Rodríguez-U., Rivera, M., Saucedo, E., Rojas, Y., Valdivia, N., Cabanillas, Q., \& Blanco, H. (2010). Parasitosis intestinales y factores socio-sanitarios en niños del área rural del distrito de Los Baños del Inca, CajamarcaPerú. Revista Médica Herediana, 21(2), 107-109. Recuperado en 24 de mayo de 2019, de http://www.scielo.org.pe/scielo. php? script $=$ sci arttext\&pid $=\mathrm{S} 1018-130 \times 20$ $\underline{10000200010 \& \operatorname{lng}=\mathrm{es} \& \mathrm{t} \operatorname{lng}=\mathrm{es}}$.

Román, R., Abril, E., Cubillas, M., Quihui, L., \& Morales, G. (2014). Aplicación de un modelo educativo para prevenir parasitosis intestinal. Estudios sociales (Hermosillo, Son.), 22(44), 92-117. Recuperado en 24 de mayo de 2019, de http://www.scielo.org.mx/ scielo.php?script $=$ sci arttext\&pid $=\mathrm{S} 0188$ 45572014000200004\&lng=es\&tlng=es.

Santa María, R. (2008). La iniciativa de vivienda saludable en el Perú. Revista Peruana de Medicina Experimental y Salud Publica, 25(4), 419-430. Recuperado de http://www.scielo.org. pe/scielo.php?script $=$ sci abstract\&pid $=\mathrm{S} 1726-$ 46342008000400013\&lng=es\&nrm=iso\&tlng $=\mathrm{es}$

Wang, X., Zhou, G., Zeng, J., Yang, T., Chen, J., \& Li, T. (2018). Effect of educational interventions on health in childhood: a metaanalysis of randomized controlled trials. Public Health, 164, 134-147. https://doi. org/10.1016/j.puhe.2018.04.013

Werner, B. (2014). Infecciones por parásitos más frecuentes y su manejo. Revista Médica Clínica Las Condes, 25(3), 485-528. https:// doi.org/10.1016/S0716-8640(14)70065-3 